\title{
EVALUATION OF PROPERTIES OF THE LASER MODIFIED SURFACE LAYER
}

\author{
A. BIEŃ \\ Machinery Engineering and Materials Dept., Agro-Technical University \\ M. Oczapowskiego 11, 10-735 Olsztyn-Kortowo, Poland \\ AND W. SZaCHNOWSKI \\ Strength and Dynamics Dept., Institute of Aviation \\ Al. Krakowska 110/114, 02-256 Warszawa, Poland
}

\begin{abstract}
This research concerns a surface layer formed on 21H12NMFA steel with a continuous wave $6 \mathrm{~kW} \mathrm{CO} 2$ laser. The purpose of the study was the determination of the physical properties of the layer with chosen methods of identification. Electromagnetic method with a point probe has been used to determine the homogeneity in numerous points of the layer. Optic and electron (TEM) microscopy enabled the identification of the microstructure of the layer, whereas using Hanemann's meter the microhardness on the surface of the sample, and also its distribution in the layer, could be evaluated. The complex characteristic of the laser formed surface layer based on the results of the above methods of investigation is presented. The study has shown that the electromagnetically determined homogeneity of the surface layer (i.e. concerning its permeability and permittivity) can be also related to thickness, structure, and microhardness of the layer.

PACS numbers: 81.40.-z, 81.65.-b, 81.70.Fy
\end{abstract}

\section{Introduction}

Usually, the area of the surface layer which one is going to modify considerably exceeds the cross section of the laser beam. In such a situation the modification of the surface layer is performed by multiple passages of the laser beam [1]. The trajectory on which the laser beam is moved notably influences the physical properties of the layer created. A surface layer resulting from the multiple passages of the laser beam displays characteristic properties which are different from those obtained with conventional surface treatments. Evaluation of such a layer calls for a lot of precision and application of different research methods because the interaction between laser beam and microzone of a material surface results in so-called "segment shaping" of the layer. Therefore, the application of fast and non-destructive methods for evaluation of the homogeneity of the surface layer created seems to be necessary. This leads to elimination of the specimens with non-uniform surface layers from subsequent research. 


\section{Experiment}

In the course of the study, the specimens made of steel grade $21 \mathrm{H} 12 \mathrm{NMFA}$ measuring $20 \times 40 \times 75 \mathrm{~mm}$ have been cut out from the heat treated $25 \mathrm{HRC}$ bar in such a manner that the longest edge was parallel to the rolling direction. The surfaces of the specimens destined for laser beam treatment were regrounded [2]. The surface layer was modified with a laser beam of rectangular cross section generated by a $\mathrm{CO}_{2}$ laser machine of $6 \mathrm{~kW}$ continuous rating*. During the course of modification of the surface layer, the beam movement followed a trajectory of the "zigzag" type shown in Fig. 1. Evaluation of the modified surface layers was performed in

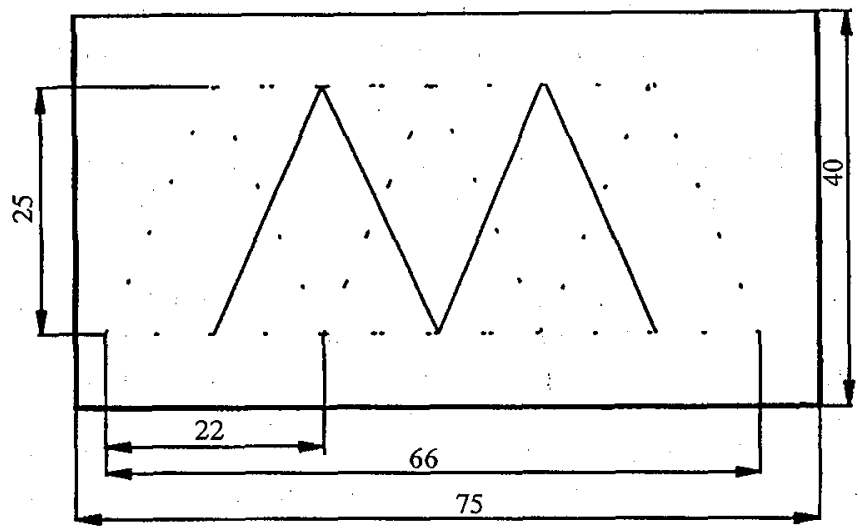

Fig. 1. Trajectory of the laser beam movement, all numbers are in $\mathrm{mm}$.

a non-destructive manner with an electromagnetic method which is based on a change in impedance of the point probe moved over the surface of specimen ${ }^{\dagger}$. The frequency of the probe has been chosen in such a way that the deepness of layer penetration was up to $\sim 1.5 \mathrm{~mm}$. Before the measurements were performed, the surface was smoothed to get rid of some local pits and other irregularities which could have possibly originated during the laser treatment course and which could have possibly introduce some undesirable scatter. Based on these measurements, a computer reconstruction of the surface layer was performed: reconstructed pictures are shown in Figs. 2 and 3 [3]. The same method of homogeneity evaluation has been applied in analytic and also experimental optimisation of parameters of the surface layer modification process. Such optimisation makes it possible to estimate the parameters of the process, which are responsible for repeatable creation of surface layers of uniform properties [1]. Selected specimens with surface layer of the assumed level of homogeneity, Figs. 4 and 5, have been exposed to metalographic examinations. Metalographic research was performed with an optic microscope, Neophot 32. Metalographic samples cut perpendicularly to the surface of the laser path and etched were examined with magnifications of $125 \times$ and $250 \times$. The second

*The laser treatments have been performed at the Technical University of Kielce, Poland.

$\dagger$ Electromagnetic examinations have been performed at the Institute of Aviation, Warsaw, Poland. 

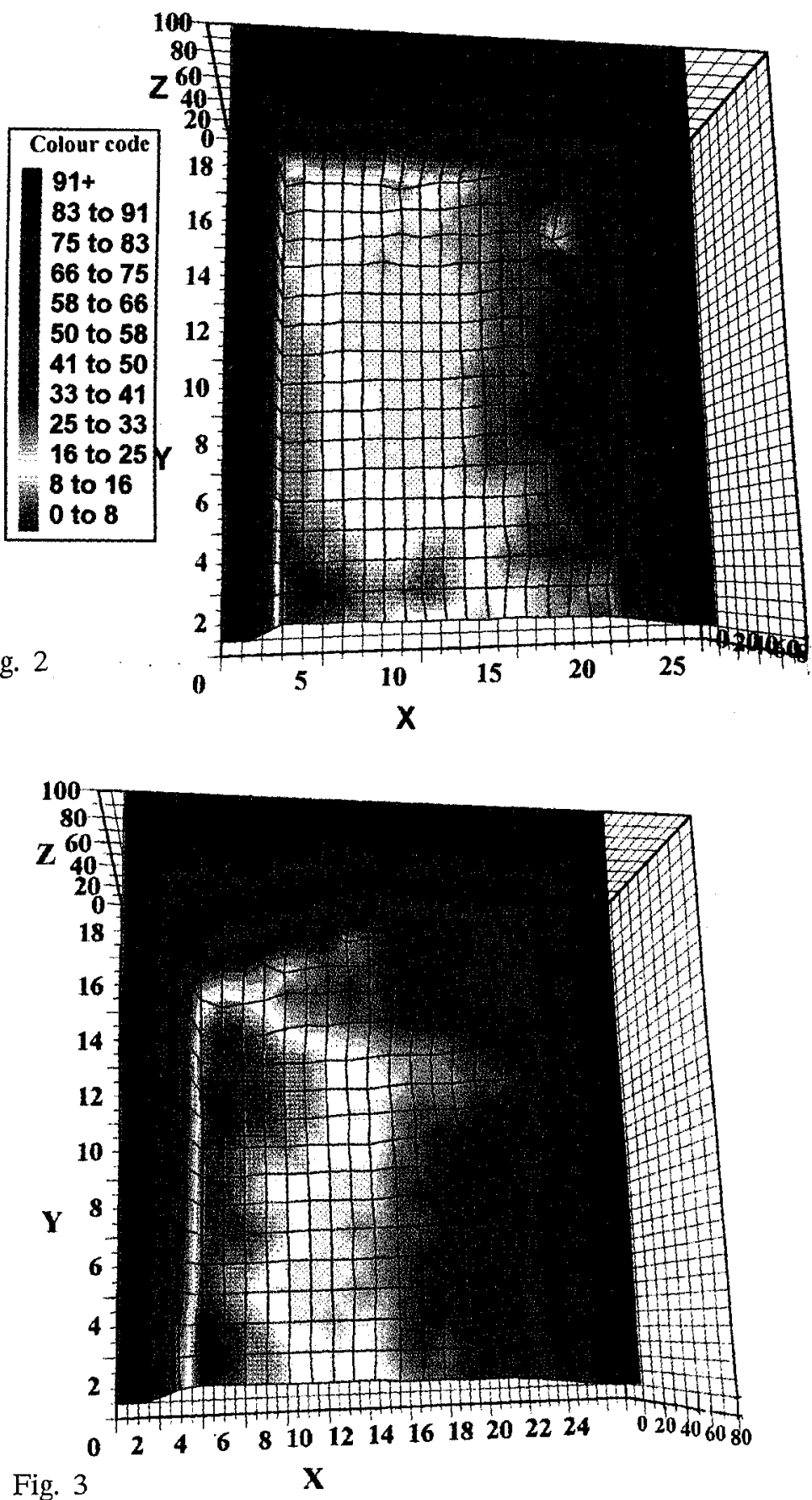

Fig. 2. Laser modified surface layer reconstructed with electromagnetic measurements. Specimen No. $10, X, Y, Z$ in $\mathrm{mm}$.

Fig. 3. As in Fig. 2, for specimen No. 9. 


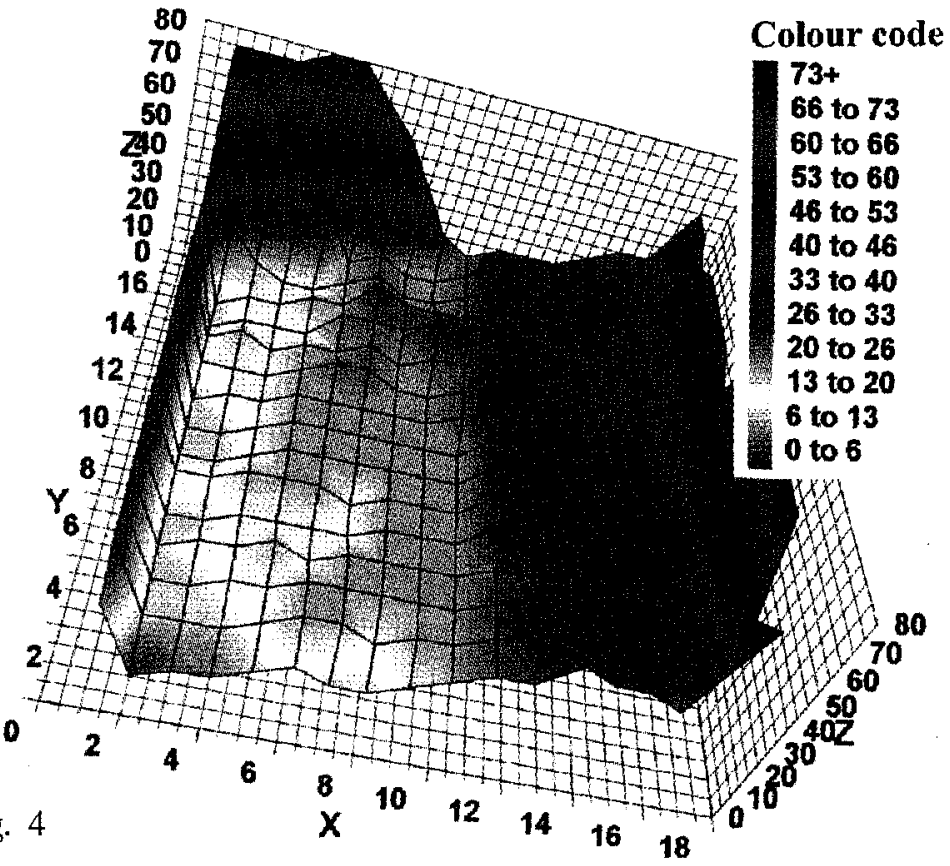

Fig. 4

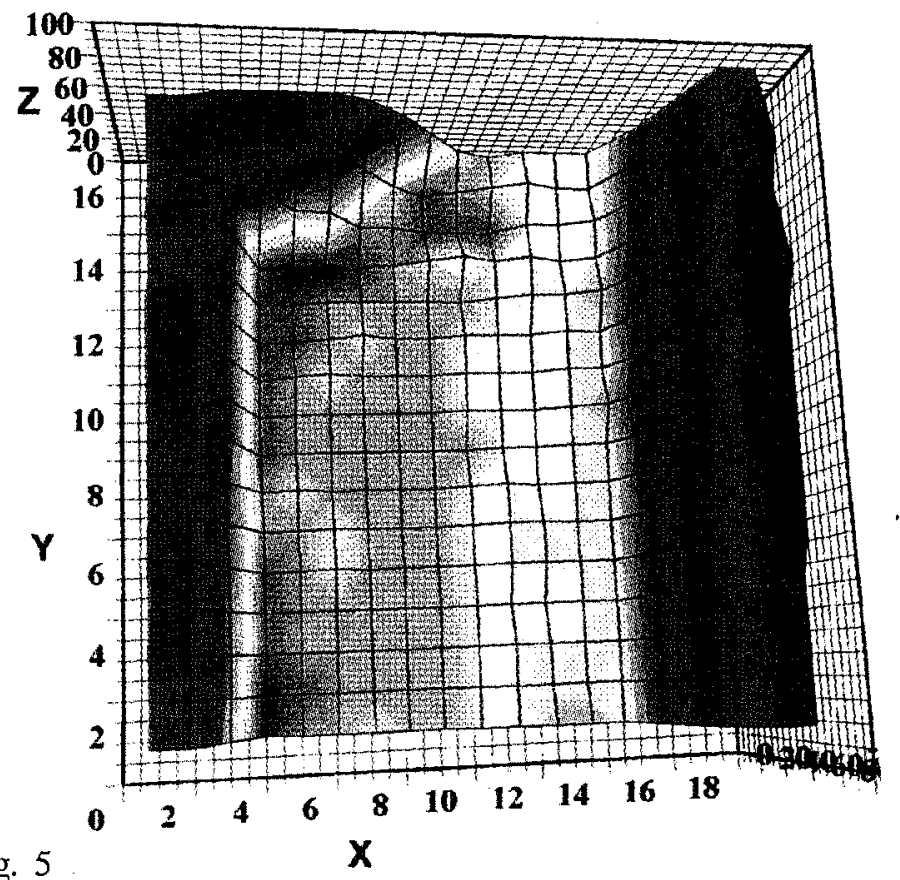

Fig. 4. Reconstructed layer of specimen No. 14, which was metalographicaly examined, $X, Y, Z[\mathrm{~mm}]$.

Fig. 5. As in Fig. 4, for specimen No. 15. 
step of metalographic examinations was performed with a transmission electron microscope, TEM type TMF $100 \mathrm{C}$. The foil with a parallel cut of $0.3 \mathrm{~mm}$ below the surface of the specimen was examined at magnifications of $6000 \times$ to $60000 \times$ and the electron diffraction method of phase identification was performed ${ }^{\ddagger}$. The microhardness measurements and its clistribution over the layer were performed with Hanemann's attachment to the optic microscope at $100 \mathrm{~g}$ loading $\$$.

\section{Results}

\subsection{Optic microscopy}

Examination of the samples revealed that the surface layer had been penetrated to the depth required. Figures 6 and 7 show a general view cross section of the laser modified layer. There are three essential characteristic zones from the laser processing point of view: the bed (Fig. 6, point 1), the penetration zone created by double passage of the laser beam (Fig. 6, point 2), and the path contact created by two adjacent passages of the laser beam (Fig. 6, point 3 ). The bed zone

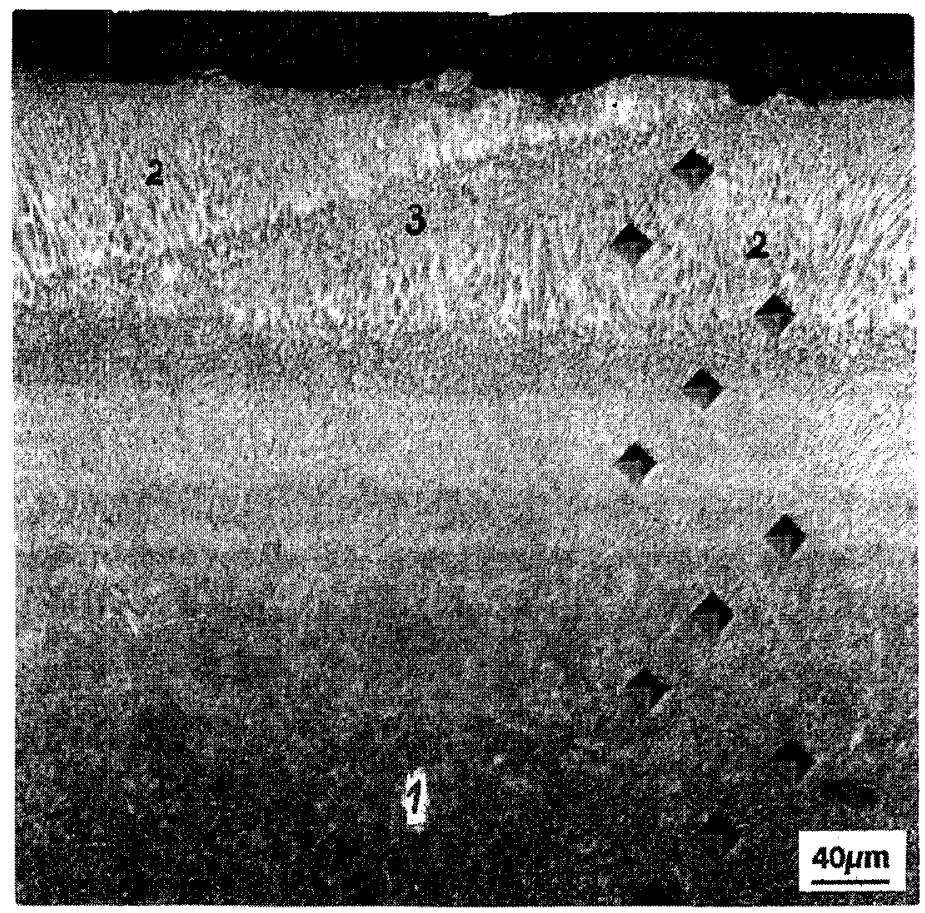

Fig. 6. Macrostructure of the layer of specimen No. 14, 1 - structure of the matrix tempered martensite, 2 - double melted zone - dendritic structure, 3 - the contact zone - frozen crystals.

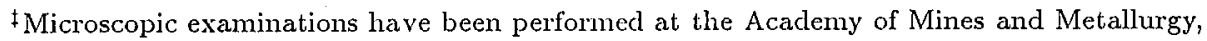
Cracov, Poland.

${ }^{\S}$ Measurements of microhardness have been performed at the Agro-Technical University, Olsztyn, Poland.
} 

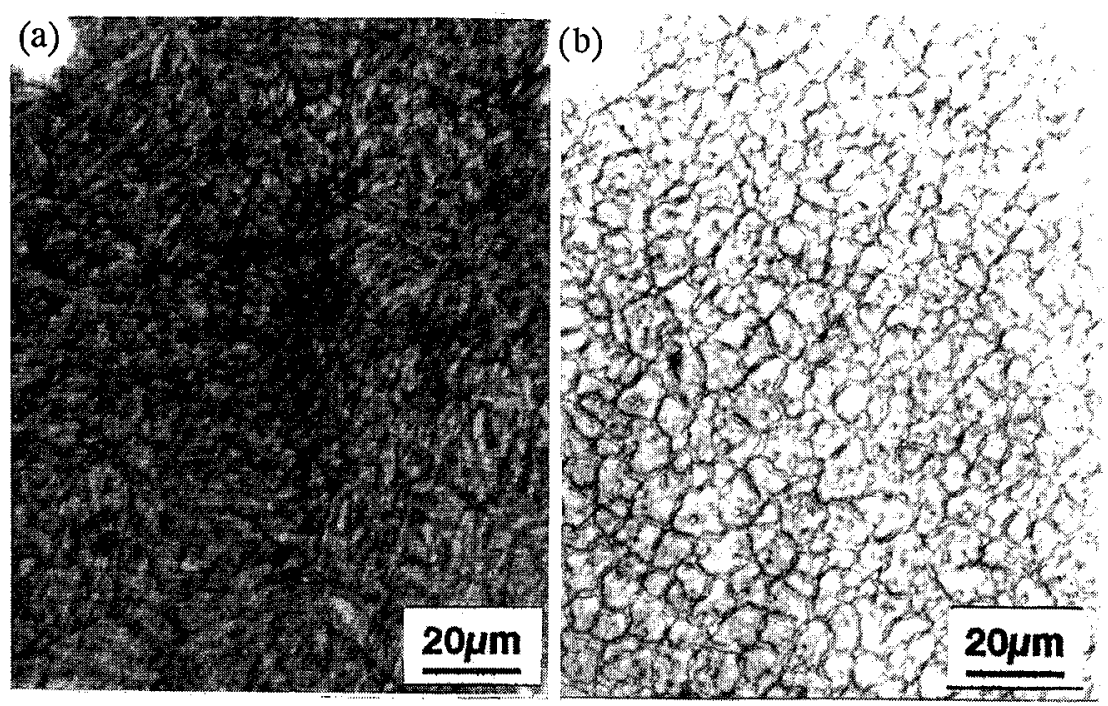

Fig. 7. Blow up of Fig. 6 at: (a) point 1, (b) point 3.

of martensite structure contains mostly tempered martensite. In the penetration zone, which is melted due to a double passage of the laser beam, the dendritic structure is dominant. The third zone, resulting from the contact of two paths, is a frozen crystal area.

\subsection{Electron microscopy}

The electron microscope (TEM) investigations were performed on foils prepared with samples drawn from the same sources supplying the samples for the optic microscopy, i.e. from the bed, double melted, and contact zones, Figs. 8,

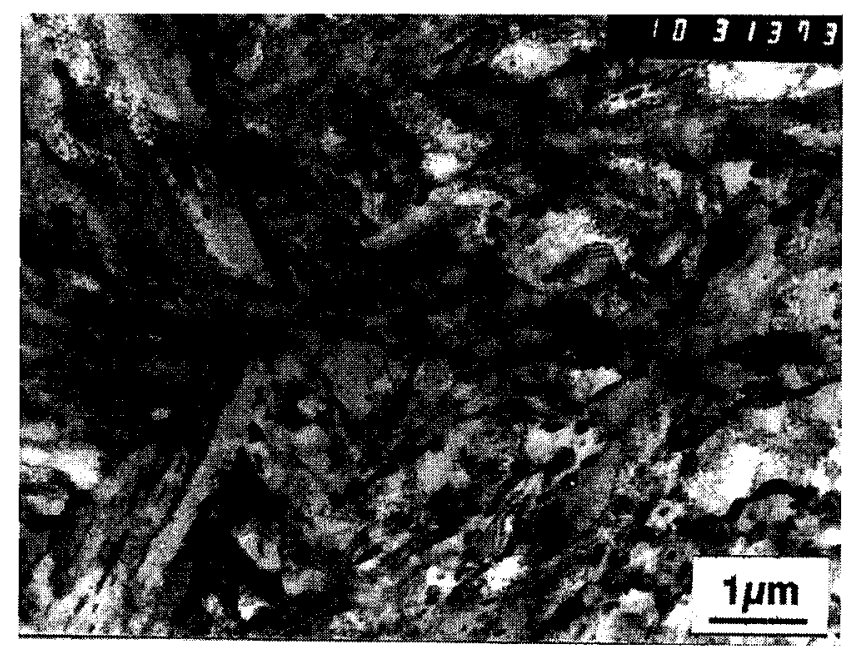

Fig. 8. Microstructure of the matrix of specinen No. 14 - tempered martesite. 

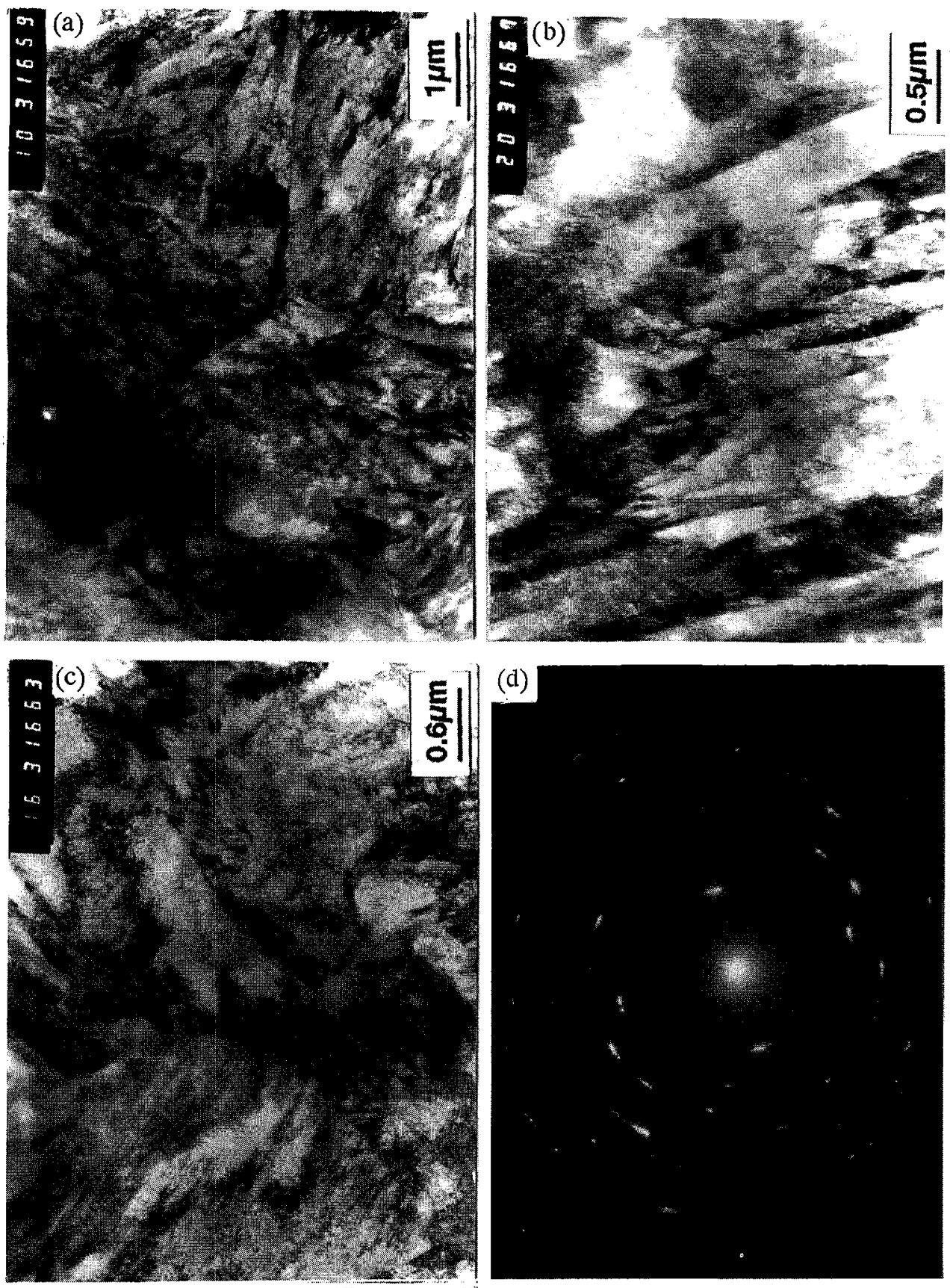

(d)

Fig. 9. Microstructure of a double melted zone of specimen No. 14, (a) tempered martensite, fine needle-shaped highly defected martensite, dislocations, dendritic segregation, (b) martensite lath, (c) martensite plates, (d) diffraction pattern. 


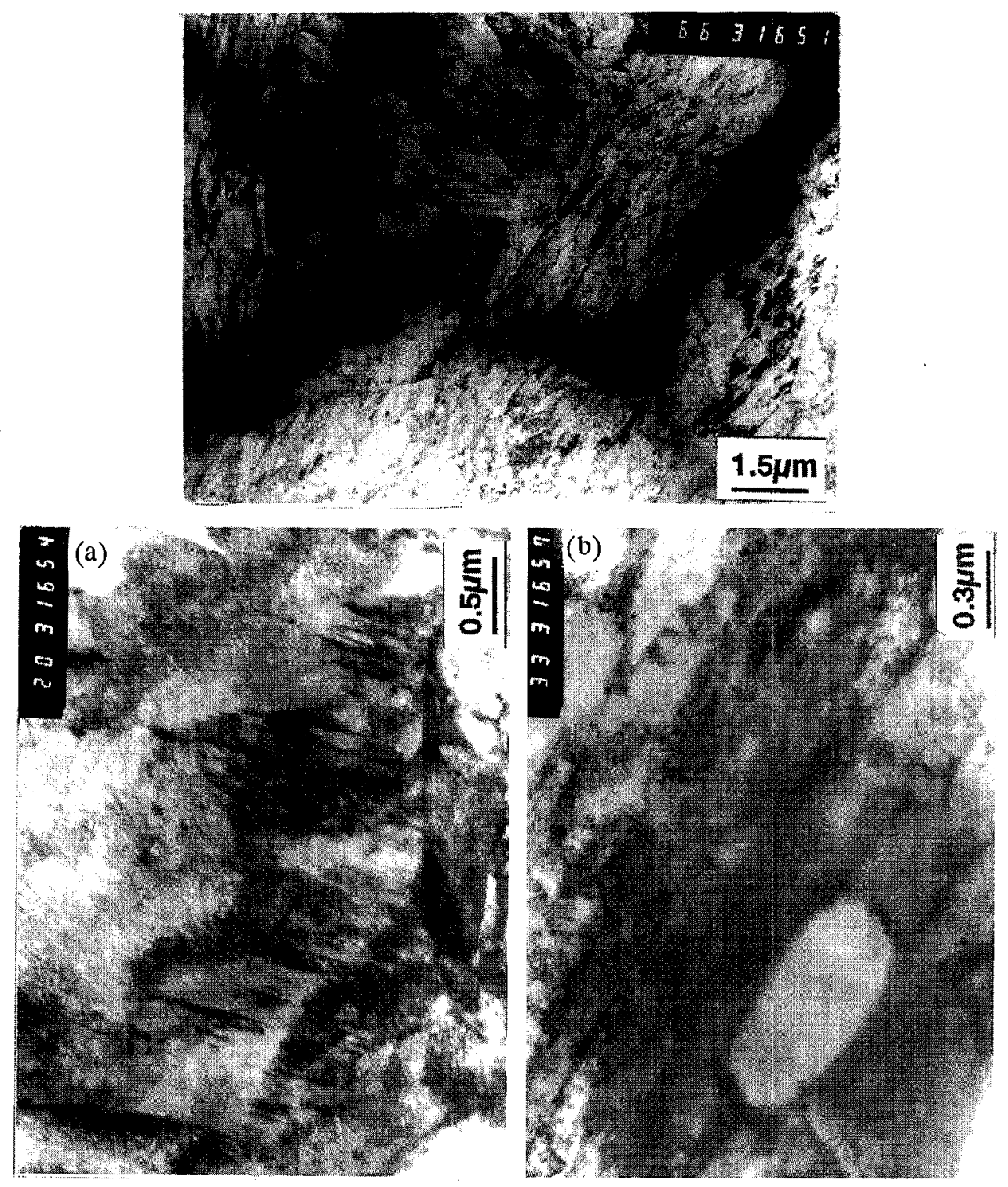

Fig. 10. Microstructure of the contact zone of specimen No. 14 - refined martensite and segregations; (a) Highly twinned martensite structure; (b) $\delta$-ferrite and carbides inclusions.

9 and 10. Figure 8 presents TEM micrography of the bed that shows mainly tempered martensite with carbide, $\mathrm{M}_{23} \mathrm{C}$, precipitates. Carbide precipitates relate as well to martensite lath boundaries as to former austenite grain boundaries and also to intragrains of former austenite. The micrography of the double melted zone, Fig. 9, shows fine, highly defective martensite with a high number of dislocations. 
Further examinations of this zone, at higher magnification, made it possible to identify the fine, highly defective tempered martensite dislocations and dendritic segregations, Fig. 9a, martensite lath structure, Fig. 9b and, martensite plates, Fig. 9c, which occur in the case of an unstable chemical composition (i.e. lack of chemical homogeneity). Figure $9 \mathrm{~d}$ shows the diffraction pattern of a sample drawn from the double melted zone. The broadening of reflections reveals a considerable disorientation existing in this zone. The contact zone, Fig. 10, shows an existence of frozen crystals, which are a more refined martensite structure than in the case of the double melted zone. It is mostly lath maitensite. One can see also sharply evident dendritic segregation regions. The highly twined martensite is seen at a higher magnification, Fig. 10a, and, in Fig. 10b, an area containing $\delta$-ferrite and $\mathrm{M}_{23} \mathrm{C}$ carbide precipitation is shown.

\subsection{Microhardness}

Homogeneity of the surface layer can be evaluated with the help of computer reconstruction. With this technique the pictures of the surface layer for specimen No. 14 have been reconstructed in Figs. 11 and 12. Figure 11 shows the pictures reconstructed on the base of electromagnetic measurements and, in Fig. 12 pictures of

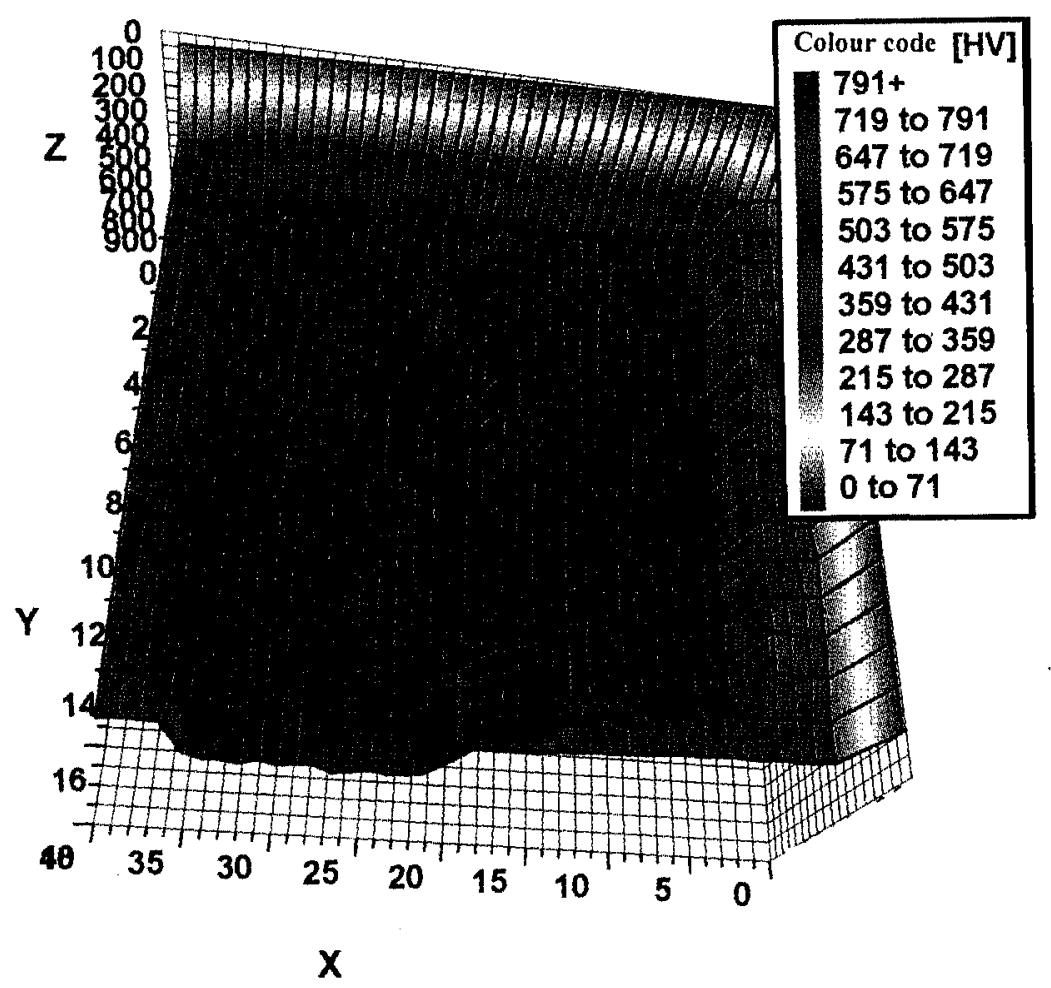

Fig. 11. Distribution of microhardness - reconstruction of the layer of specimen No. $15, X, Y, Z[\mathrm{~mm}]$. 


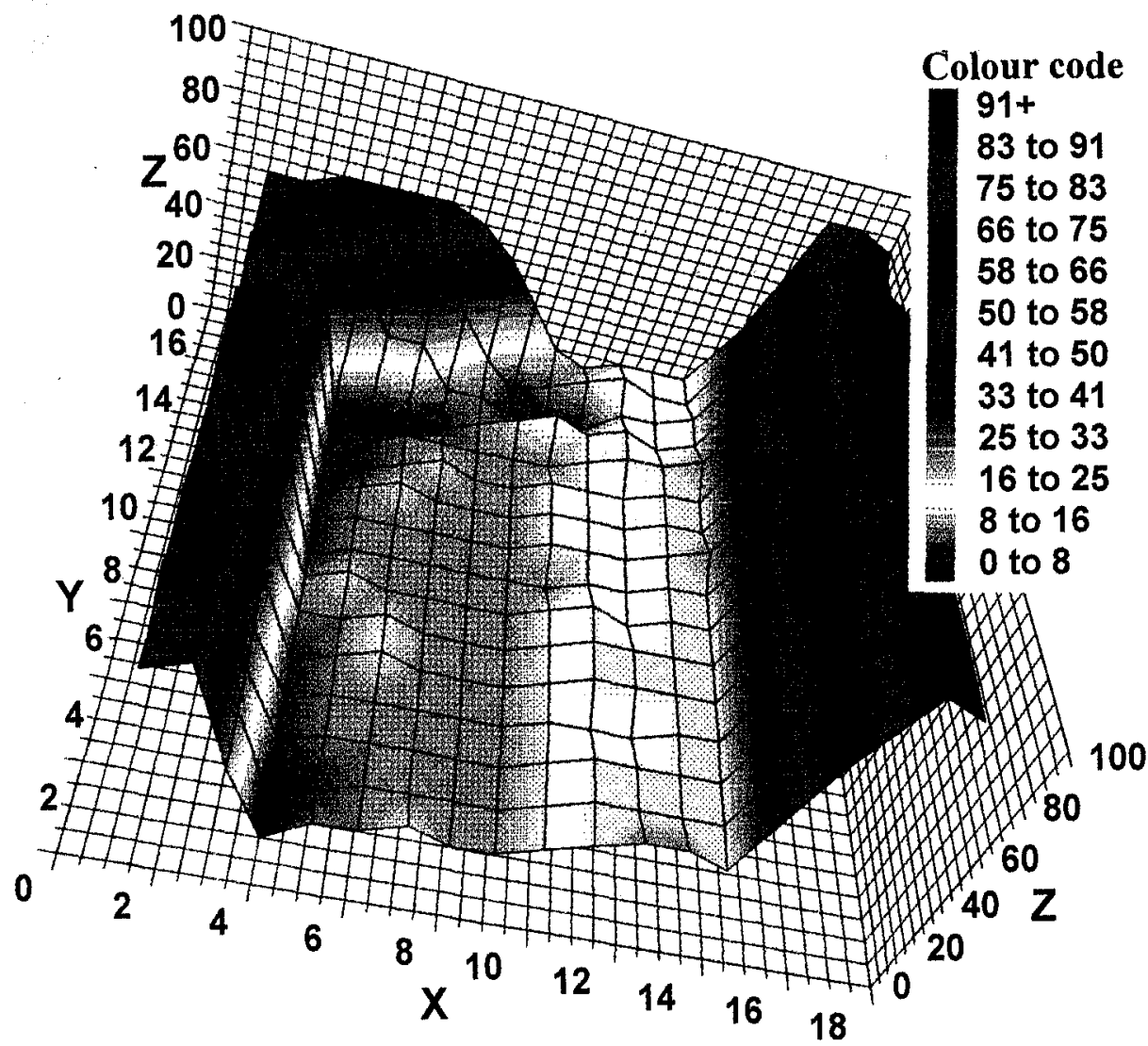

Fig. 12. Distribution of microhardness - reconstruction of the layer on the electromagnetic measurements base, specimen No. 15, $X, Y, Z[\mathrm{~mm}]$.

the same surface layer reconstructed on the base of microhardness measurements. The layer homogeneity was found to be $85 \%$ and $88 \%$, evaluated respectively on the base of electromagnetic and microhardness measurements results.

\section{Conclusions}

Taking into account the crystal structure, electromagnetic, and also microhardness measurements, the laser modified suriace layer can be considered homogeneous. This homogeneous layer exhibits a frequent repetition of structure segments, i.e. melted and contact zones. The segmentation results from an accepted technology of surface layer modification. Optimisation of laser parameters for creation of repeatable layers follows the procedure established by the authors $[1,2,4]$. Up to now the optimisation has been experimentally proved with electromagnetic, microhardness, and some metalographic investigation. As a result of the homogeneity analysis the quadratic (encoded) equation with iterations has been computed [1].

The equation represents a magnitude of homogeneous surface as a function of power, $X_{1}$, and displacement velocity, $X_{2}$, and, width of the laser beam, $X_{3}$. 
The laser modification of the layer results in hardened material better than that conventionally hardened. The hardness level for a laser treated steel ranges above HV1200 whereas in the case of a conventional hardening up to HV800. Both the doubly melted and contact zones reveal a higher microhardness than the substrate. Microhardness measurement results show a uniform distribution over the surface of the specimens. Some scatter between those two homogeneity evaluations, i.e. $88 \%$ for electromagnetic and $85 \%$ for microhardness, that is not connected with the above-mentioned two zones, confirms former assumptions that a fine martensite structure is mostly responsible for that microhardness distribution [5]. In both zones the grain refinement resulting in rapid laser heating, and also the high stress level induced by cooling that refines martensite substructure, are responsible for the properties of the created layer. Although the differences in the microstructure of the two zones are evident, they do not significantly influence the macroscopic surface properties of the modified layers.

\section{Acknowledgements}

The authors wish to thank Mr. C.F. Majkrzak, Ph.D. from NIST, for his help in preparing the manuscript.

\section{References}

[1] A. Bień, W. Szachnowski, in: Proc. SPIE, Laser Technology V, Applications in Materials Science and Engineering, Vol. 3187, SPIE, The International Society for Optical Engineering, Washington 1997, p. 48.

[2] A. Bien, to be published.

[3] W. Szachnowski, to be published.

[4] A. Bień, Ph.D. Thesis, Technical University of Gdańsk, Gdanisk 1990.

[5] M. Eomozik, G. Czaja, W. Nowak, Bulletin of Institute of Welding in Gliwice $4(40), 47$ (1996).

[6] A. Bień, to be published. 